Historic, Archive Document

Do not assume content reflects current scientific knowledge, policies, or practices. 



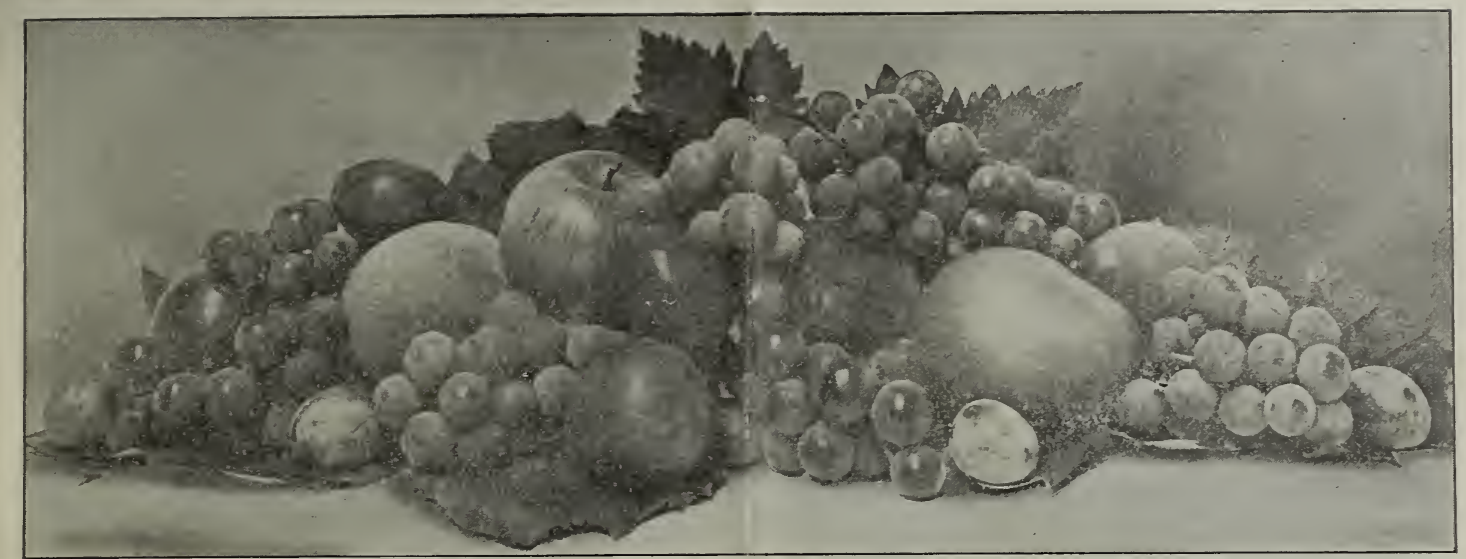

You can grow delicious fruit like this for vourself at very small expense and little trouble. Have you ever noticed the difference in flavor of fruit bought at your grocer's and the fresh fruit picked from your own trees?

\section{PRICES FOR 1913}

All Kinds of Trees, Plants, Vines, Etc.

We Make a Specialty of Growing Quality Trees.

\section{WHY OUR TREES ARE THE BEST}

Our aim for thirty-three years has been to produce only the highest quality in our trees and plants. We have spent an enormous amount of money and years of labor on our Experimental Farm, trying out new methods of growing, cultivating, etc. We have gradually improved the quality of our trees over other growers, until now our stock is recognized by the leading orchardists as the best in the country.

There is no one kind of soil that will produce the highest quality in every class of tree or plant. Each class or group of classes requires a different soil to bring the best results. For instance, the group of which apples and pears are examples, requires a heavy clay soil, while the group which includes peaches and cherries does better on a loose sandy loam.

We have several farms and on each farm we grow only that group which does best on that farm. So you see we produce in each tree or plant the highest quality that is possible to produce. To begin with we have the finest land to grow on. Then we use only the finest seedling stock to bud or graft our trees on. All our buds and scions are selected from bearing trees in our own orchards. IVe feed our trees all the fertilizer they will take. We cultivate all of our farms with as much care as if they were gardens. And most important of all-the men who are growing these trees for us have been with us for the past twenty-five or thirty years, and are now experts in tree and plant growing. Some of our men have sons who were born and have been brought up on our nurseries and who know the business of growing from top to bottom. They are experts in their respective departments. Don't overlook this important factor.

If you want quality stock at a reasonable price you had better give us a trial. We have a catalog giving complete description and prices of everything we have to offer. If you have not a copy send us a postal card and we will mail you one.

\section{Green's Nursery Co., Rochester, N. Y.}

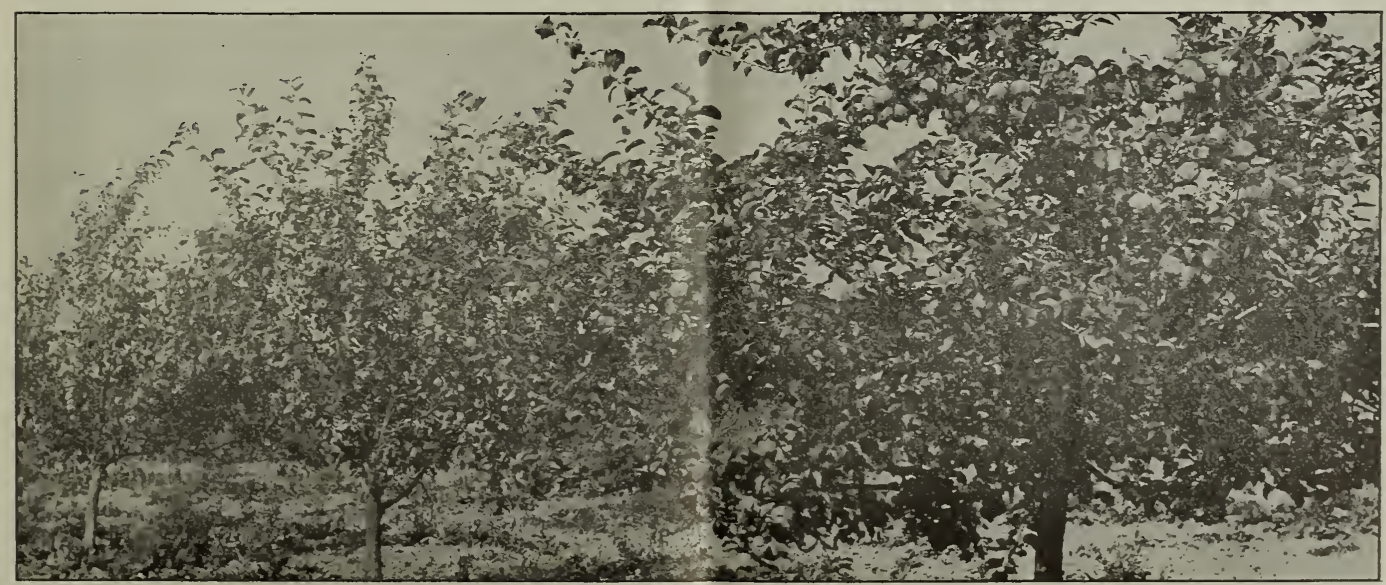

If you look closely at this illustration you will notice that the trees are loaded with beautiful apples. Do you realize that a small orchard of apple trees like this would take care of you in your old age, or a few trees not only supply you with what you want to eat but enable you to buy a few luxuries that you feel you cannot afford now? 


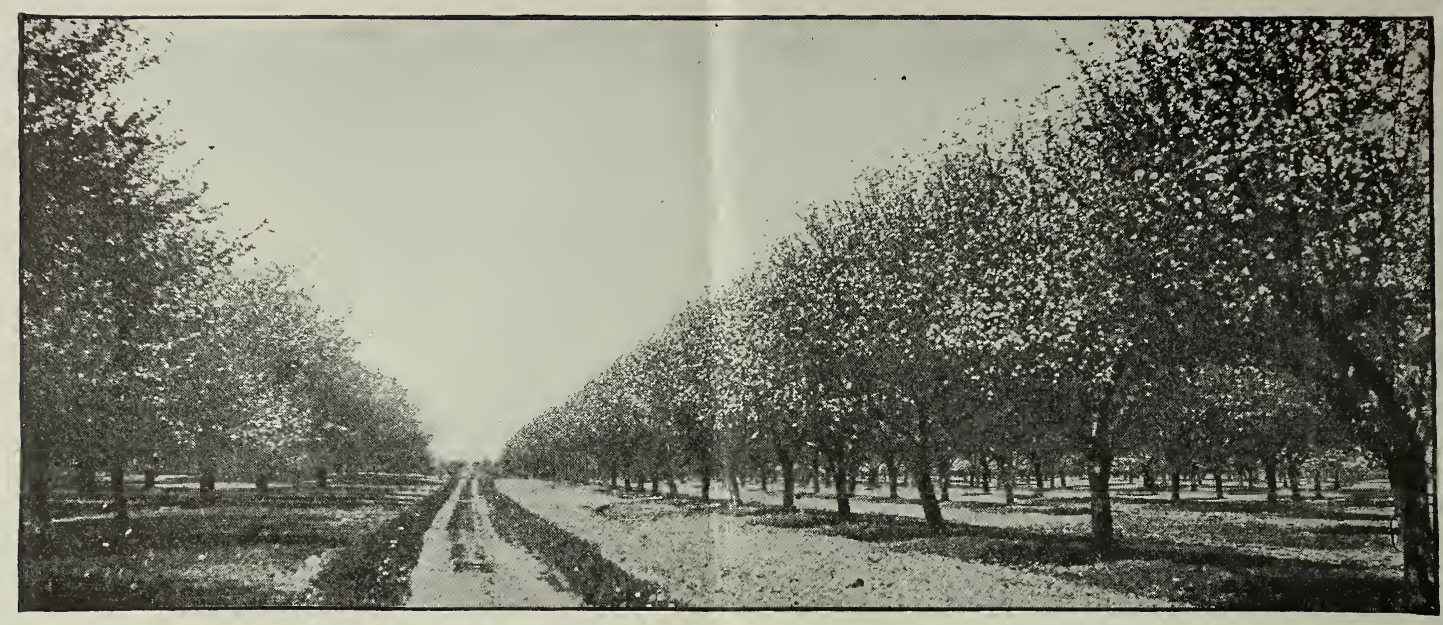

A view of the Collamer Apple Orchard at Hilton, N. Y. Note the clean cultivation.

\section{APPLES}

Prices of General Varieties-not Starred* Two to Three Year Apple Trees

$\begin{array}{rrr}\text { First Class Largest or Extra Size.. } \$ 0.35 & \$ 10 & 100 \\ \text { First } & \text { Each } & \$ 25.00\end{array}$ $\begin{array}{lrrr}\text { First Class Medium or Orchard Size } & .30 & 2.50 & 20.00 \\ \text { First Class Smaller Size, Branched } & .25 & 1.75 & 15.00\end{array}$ Prices of Starred* Varieties, Rare and Valuable,
Superior Quality

$\begin{array}{rrr}\text { Each } & 10 & 100 \\ \text { First Class Largest or Extra Size.. } \$ 0.40 & \$ 3.50 & \$ 30.00\end{array}$ $\begin{array}{lrrr}\text { First Class Medium or Orchard Size } & .35 & 3.00 & 25.00 \\ \text { First Class Smaller Size, Branched } & .25 & 2.00 & 17.50\end{array}$ ALPHABETICAL LIST OF APPLE TREES
Alexander

Alexander

Arkansas Beauty

* Baldwin

Bayard

Bellfower.

Ben Davis

Ben Hu

Black Ben Davis

Blenheim Orange

Canada Red

Chenango Strawberr

Duchess of Oldenburs

Fameuse

Gano

Gravenstein

Green's Baldwi

Grimes Golden
Hubbardston

Hyslop Crab

Jonathan

Maiden's Blush

McIntosh Red

Newtown Pippin
North Star

\section{DWARF APPLES}

Two to Three Year Dwarf Apple Trees

First Class Well Branched ....... $\$ 0.40 \quad \$ 3.50$

$$
\text { LIST OF DWARF APPLES }
$$

\begin{tabular}{ll} 
Alexander & Red Astrachan \\
Baldwin & R. I. Greening \\
Duchess of Oldenburg & Twenty Ounce \\
Fameuse & Wealthy \\
Gravenstein & Yellow Transparent \\
\hline
\end{tabular}

\section{PLUMS}

Two to Three Year Old Plum Trees

$\begin{array}{lllrr} & & \text { Each } & 10 & 100 \\ \text { First Class Largest } & \text { Size ......... } \$ 0.30 & \$ 2.50 & \$ 22.50 \\ \text { First Class Medium } & \text { or Orchard Size } & .25 & 2.00 & 17.50 \\ \text { First Class Smaller Size, Branched } & .20 & 1.50 & 12.50\end{array}$

LIST OF TWO TO THREE YEAR PLUM TREES

$\begin{array}{ll}\text { Abundance, Red } & \text { Ogon, Yellow } \\ \text { Apple, Red } & \text { Peter's Yellow Gage, Yellow } \\ \text { Bradshaw, Red } & \text { Pond's Seedlings, Red } \\ \text { Burbank, Red } & \text { Purple Egg, Red } \\ \text { Climax, Red } & \text { Red Egg, Red } \\ \text { Coe's Golden Drop, Yellow } & \text { Red June, Red } \\ \text { German Prune, Blue } & \text { Reine Claude, Yellow } \\ \text { Grand Duke, Blue } & \text { Satsuma, Reddish } \\ \text { Gueii, Black } & \text { Shipper's Pride, Blue } \\ \text { Imperial Gage, Yellow } & \text { Shropshire Damson, Blue } \\ \text { Lombard, Red } & \text { Thanksgiving Prune, Blue } \\ \text { Monarch, Blue } & \text { Wickson, Blue } \\ \text { Moore's Artic, Blue } & \text { Yellow Egg, Yellow } \\ \text { Niagara, Red } & \text { York State Prune, Blue }\end{array}$

Price of One Year Old Plum Trees

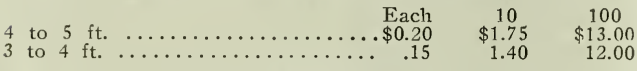

Burbank

VARIETIES

\section{CHERRIES}

Prices of All Varieties of Cherry Trees, Two to Three Year

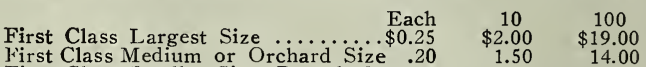

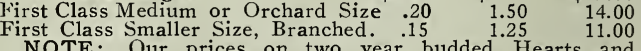
NOTE: Our prices on two year budded Hearts and Bigarreaus (sweet) and Dukes and Morellos (sour) ar
the same.

LIST OF TWO TO THREE YEAR CHERRY TREES Duke and Morello Red Type (Sour)

$\begin{array}{ll}\text { Dyehouse } & \text { May Duke } \\ \text { Early Richmond } & \text { Montmorency } \\ \text { English Morello } & \text { Ostheim } \\ \text { Late Duke } & \text { Olivet }\end{array}$

Louis Phillippe

Hearts and Bigarreaus Type (Sweet)

Centennial, White $\quad$ Napoleon, White

Green's Tartarian, Blac

Black Tartarian, Black

Yellow Spanish, Yellow Prices of One Year Cherry Trees
(On Mazzard roots)

Hirst Class, Branched $\ldots \ldots \ldots \ldots . \$ 0.25 \quad \begin{array}{ll}\text { Each } & 10 \\ & 1.75\end{array}$

Varieties of One Year Cherry Trees

$\begin{array}{ll}\text { Bing } & \text { Napoleon } \\ \text { Green's Tartarian } & \text { Windsor }\end{array}$

NOTE: Our one year cherries are all on Mazzard roots

\section{STANDARD PEAR TREES}

Prices of General Varieties (except Bartlett
and Worden Seckel)

\begin{tabular}{rrr} 
First Class Largest or Extra Size .. $\$ 0.30$ & $\$ 10$ & 100 \\
\hline 2.75 & $\$ 25.00$
\end{tabular} $\begin{array}{lrrr}\text { First Class Medium or Orchard Size } & .25 & 2.25 & 20.00 \\ \text { First Class Smaller Size, Branched .. } & .20 & 1.75 & 12.50\end{array}$ PRICE OF BARTLETT STANDARD PEARS. $\begin{array}{lrrr} & \text { Each } & 10 & 100 \\ \text { First Class Largest Size .......... \$0.35 } & \$ 3.25 & \$ 30.00 \\ \text { First Class Medium or Orchard Size } & .30 & 2.75 & 25.00 \\ \text { First Class Smaller Size, Branched ... } & .25 & 2.25 & 17.50\end{array}$

LIST OF STANDARD PEAR TWO TO THREE

$\begin{array}{ll} & \text { YEAR TREES } \\ \text { Anjou } & \text { Lawrence } \\ \text { Bartlett } & \text { Louise Bonne } \\ \text { Clapp's Favorite } & \text { Seckel } \\ \text { Flemish Beauty } & \text { Sheldon } \\ \text { Gans Early } & \text { Wilder Early } \\ \text { Kieffer } & \text { Worden Seckel }\end{array}$

\section{DWARF PEAR TREES}

First Class Largest or Extra Size.. $\begin{array}{rrr}\text { Each } & 10 & 100 \\ \$ 1.75 & \$ 14.00\end{array}$ $\begin{array}{lrrr}\text { First Class Largest or Extra Size.. } \$ 0.25 & \$ 1.75 & \$ 14.00 \\ \text { First Class Medium or Orchard Size } & .20 & 1.50 & 11.00 \\ & \end{array}$ LIST OF DWARF PEAR TWO TO THREE YEAR

Anjou
Bartlett

$\begin{array}{ll}\text { Bartlett } & \text { Lawrence } \\ \text { Clairgeau } & \text { Louise Bonne } \\ \text { Clapp's Favorite } & \text { Seckel }\end{array}$

$\begin{array}{ll}\text { Duchess } & \text { Tyson } \\ \text { Flemish Beauty } & \text { Wilder Early }\end{array}$

$\begin{array}{ll}\text { Glemish Beauty } & \text { Wilder Early } \\ \text { Worden Secke }\end{array}$

Howell

\section{QUINCES}

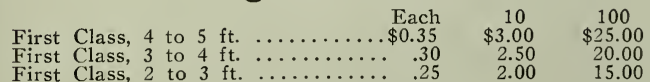
LIST OF QUINCE TREES

Bourgeat
Bing, Black

Governor Wood, White Schmidt's Bigargeau, White

Meech's Prolific 


\section{PEACHES}

Price of All Standard Peach Trees Except Niagara

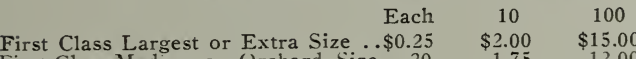
First Class Smaller Size, Branched ..

LIST OF BEST STANDARD PEACH TREES Beer's Smock

Carman

Champion

Crawford Early

Crawford Late

Erosby Rivers

Fitzgerald

Golden Drop

Hill's Chili

Mountain Ros

Sneed

Sneed St Jown

Price of Niagara Peach

$\begin{array}{lrrr} & \text { Each } & 10 & 100 \\ \text { First Class Largest or Extra Size } & \$ \$ 0.30 & \$ 2.50 & \$ 17.50 \\ \text { First Class Medium or Orchard Size } & .25 & 2.00 & 15.00\end{array}$ $\begin{array}{llll}\text { First Class Smaller Size, Branched... } & .20 & 1.50 & 12.00\end{array}$

\section{NUTS}

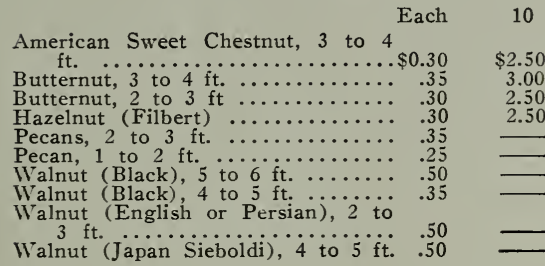

\section{GRAPES}

Strong Two Year Grape Vines, Best Varieties

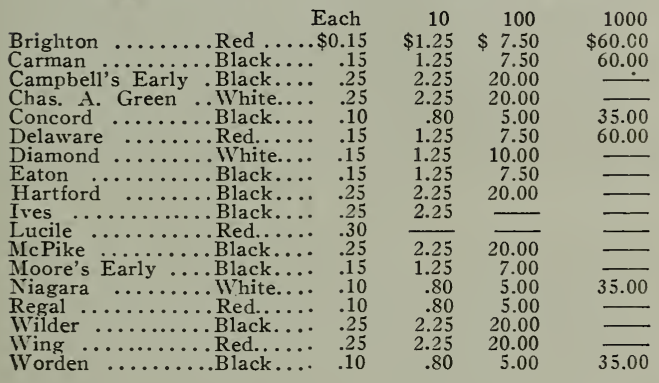

\section{GOOSEBERRIES}

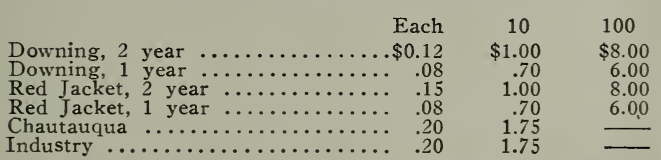

\section{CURRANTS}

Price of Two Year Old Currant Bushes No. Comet $\ldots . \ldots \ldots \ldots$ Red.... Each $\begin{array}{rrrr}10 & 100 & 1000\end{array}$

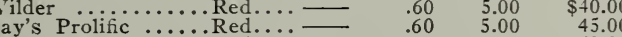

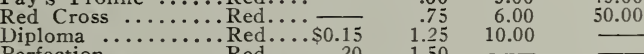

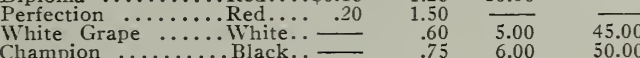
Price of One Year Old Currant Bushes No. 1

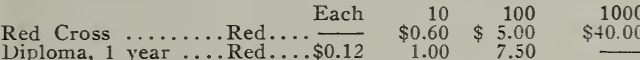

Price of Two Year Old Current Bushes No. 2

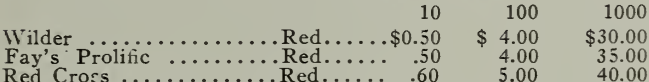
$\begin{array}{lllll}\text { Red Cross ............. Red ...... } & .60 & 5.00 & 40.00 \\ \text { White Grape ... }\end{array}$

\section{RASPBERRIES}

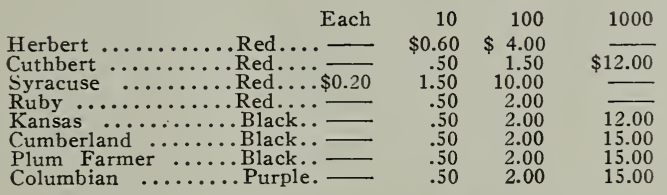

\section{BLACKBERRIES}

Eldorado $\ldots \ldots \ldots \ldots \ldots \ldots \ldots \ldots . \$ \begin{array}{rrr}10 & 100 & 1000 \\ 50 & \$ 3.00 & \$ 20.00\end{array}$

\section{STRAWBERRIES}

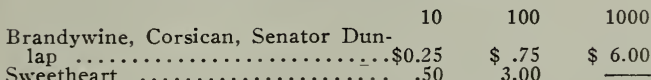

\section{GARDEN ROOTS}

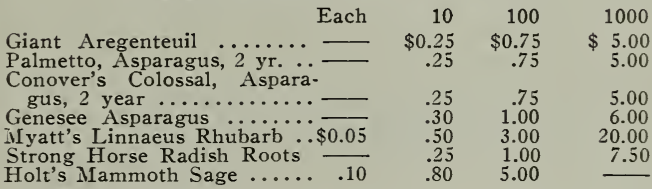

\section{EVERGREEN TREES}

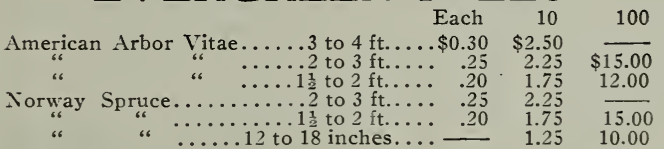

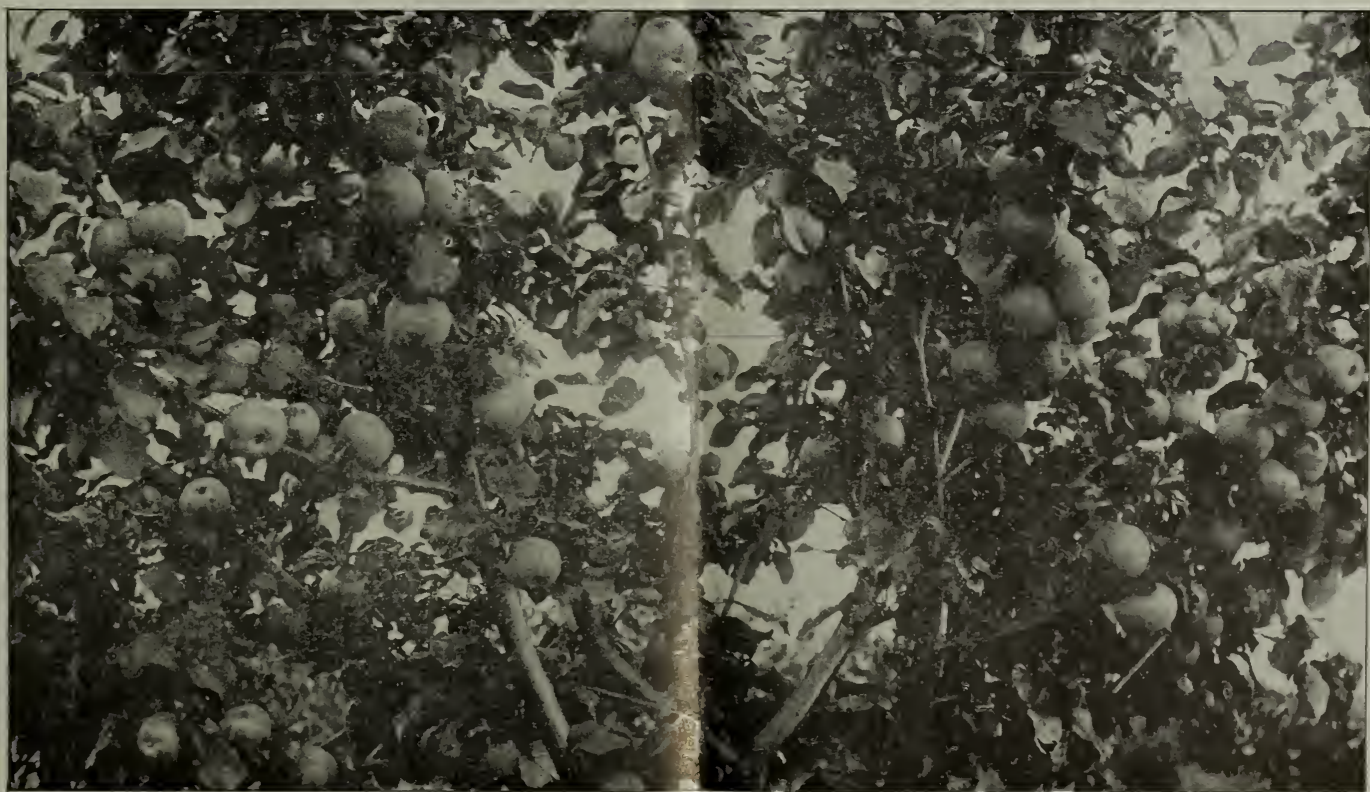

This is the way the apples grow on vigorous trees. Green's trees have always been noted for their vigor and hardiness. 


\section{ORNAMENTAL TREES}

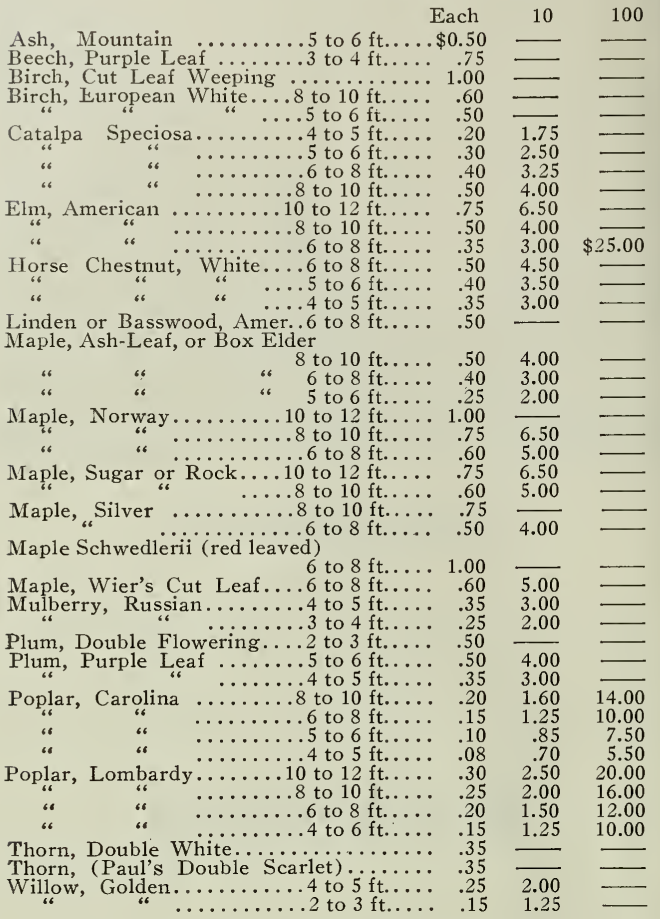

\section{HEDGE PLANTS}

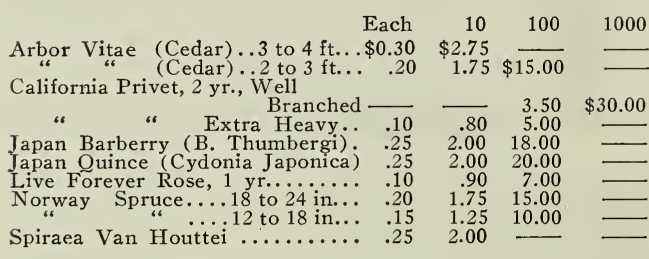

\section{FLOWERING VINES}

American Ivy (Virginia Creeper) $\ldots \ldots \ldots \ldots \ldots \ldots \ldots \ldots \ldots$ American Ivy (Virginia Creeper) $\ldots \ldots \ldots \ldots \ldots \ldots \ldots \ldots \ldots .25$
Boston Ivy (Ampelopsis Veitchii) $\ldots \ldots \ldots \ldots \ldots \ldots \ldots \ldots$ Clematis: $\left\{\begin{array}{l}\text { Henryi } \ldots \ldots \ldots \ldots \ldots \ldots \\ \text { Jackmani } \ldots \ldots \ldots \ldots\end{array}\right.$

Honeysuckle (Hall's Japan)

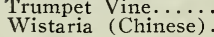

\section{ORNAMENTAL SHRUBS}

\begin{tabular}{|c|c|c|}
\hline & Each & 10 \\
\hline $\begin{array}{l}\text { Althea (Rose of Sharon) } 2 \\
\text { Barberry (Purple Leaved) }\end{array}$ & & $\$ 2.00$ \\
\hline $\begin{array}{c}\text { Barberry (Purple Leaved) } \\
\text { Cranberry Tree.......... }\end{array}$ & .2 & 2.00 \\
\hline Cranberry Tree........... & .2 & 2.00 \\
\hline Deutzia Candida (White) .... & .25 & 2.00 \\
\hline Deutzia Crenata Rose & .25 & 2.00 \\
\hline (Golden Leaved)..$\ldots \ldots \ldots \ldots \ldots \ldots$ & .25 & 2.00 \\
\hline . & .25 & 2. \\
\hline ..... & .25 & 2.00 \\
\hline 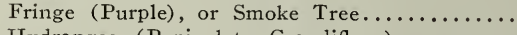 & .2 & 2.0 \\
\hline Hydrangea (Pa & $\begin{array}{l}.25 \\
.35\end{array}$ & 2.0 \\
\hline uince $\left(C_{y}\right.$ & .25 & 2.0 \\
\hline Blue, Pink $\mathrm{I}$ & .25 & 2.2 \\
\hline Lilacs, double & .35 & 3.25 \\
\hline ornia) Extra Heavy............ & .10 & .8 \\
\hline Snowball & .25 & 2.00 \\
\hline 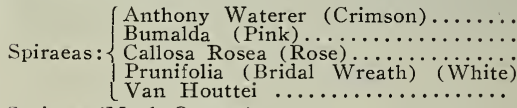 & $\begin{array}{l}.25 \\
.25 \\
.25 \\
.25 \\
.25\end{array}$ & $\begin{array}{l}2.00 \\
2.00 \\
2.00 \\
2.00 \\
2.00\end{array}$ \\
\hline (Mock Orange)..$\ldots \ldots \ldots \ldots \ldots \ldots$ & .25 & 2.0 \\
\hline Thunbergi (Dw & .25 & 2.00 \\
\hline$:\{$ & .2 & 2.0 \\
\hline
\end{tabular}

\section{HARDY FLOWERING PLANTS}

\begin{tabular}{|c|c|c|c|}
\hline & Each & 10 & 100 \\
\hline $\begin{array}{l}\text { Anchusa (Heavenly Blue Flower) New } \\
\text { Dahlias }\end{array}$ & $\$ 0.35$ & $\$ 3.00$ & \\
\hline Gladiolus & & 0.20 & $\$ \overline{1.10}$ \\
\hline Golden Glow & .05 & .40 & \\
\hline Ostrich Plume (Ornamental Grass). & .10 & .80 & \\
\hline 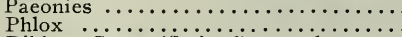 & .25 & $\begin{array}{l}2.00 \\
1.25\end{array}$ & 12.00 \\
\hline $\begin{array}{l}\text { Ribbon Grass (Striped) per clump } \\
\text { Yucca Filamentosa . ............. }\end{array}$ & .10 & .90 & \\
\hline atosa $\ldots \ldots \ldots \ldots \ldots \ldots$ & & 2.00 & \\
\hline
\end{tabular}

\section{ROSES}

Price of Hardy Rose Bushes,....... Each $\begin{array}{r}\$ 0.25 \\ \$ 2.25\end{array}$

$\begin{array}{ll}\text { Anne de Diesbach } & \text { La France } \\ \text { Baltimore Belle } & \text { Madam Plantier } \\ \text { Clio } & \text { Magna Charta } \\ \text { Coquette des Alps } & \text { Margaret Dickson } \\ \text { Coquette des Blanche } & \text { Moss (Pink, White, Red) } \\ \text { Crimson Rambler } & \text { Paul Neyron } \\ \text { Dorothy Perkins } & \text { Prince Camille D'Rohan } \\ \text { Gen. Jacqueminot } & \text { Rambler (White or Yellow) } \\ \text { Lady Gay } & \text { White Baby Rambler }\end{array}$

Prices of Special Roses

\begin{tabular}{|c|c|c|c|}
\hline & Each & 10 & 100 \\
\hline Frau Karl & & & \\
\hline ve Forever, 2 yr. $\ldots \ldots \ldots \ldots \ldots$. & .15 & 1.25 & $\$ 12.00$ \\
\hline , 1 yr... & & & 7.00 \\
\hline , . & .3 & 3.00 & \\
\hline rimson & .35 & 3.00 & 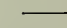 \\
\hline lcher & .35 & 3.00 & \\
\hline
\end{tabular}

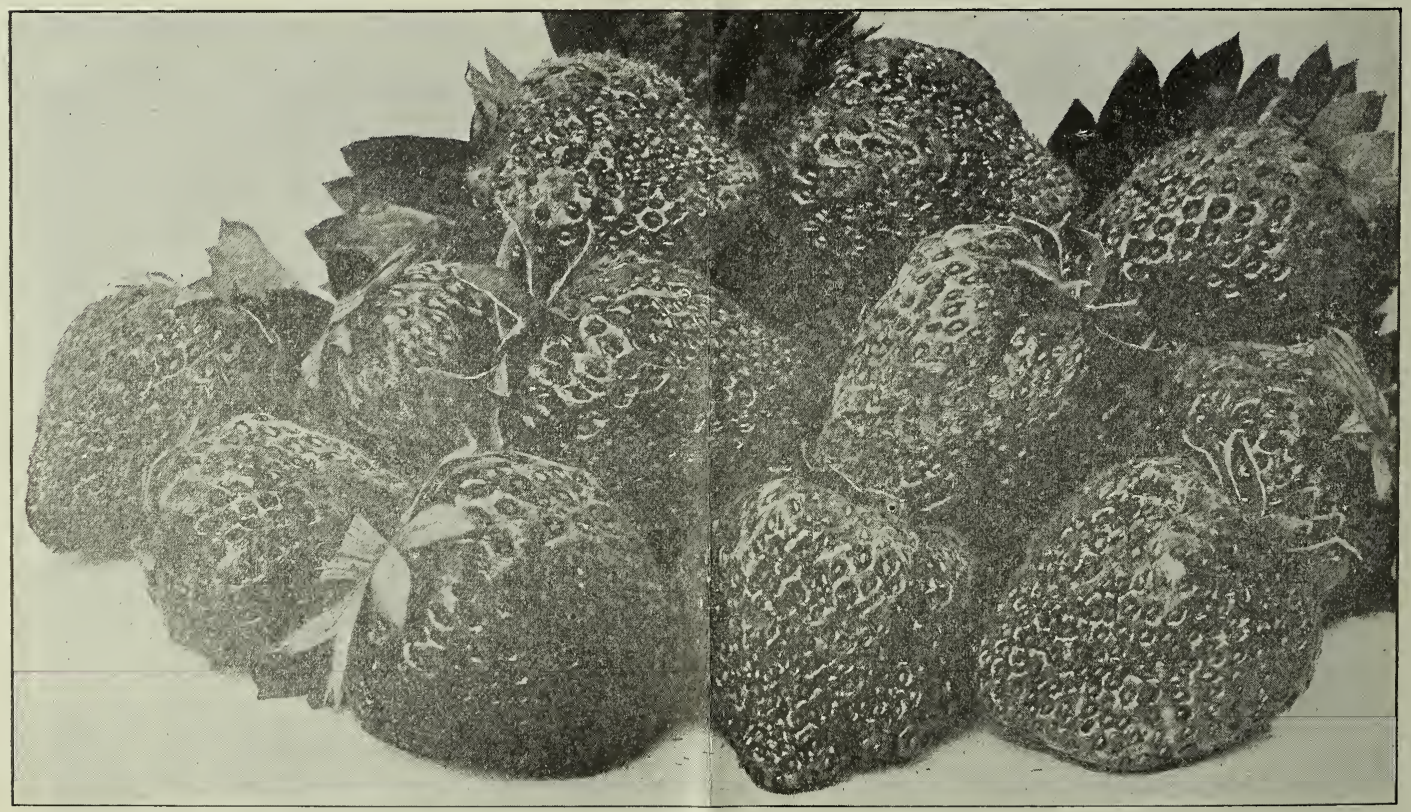

These are Sweetheart strawberries, the variety that we are offering as a premium. Prof. H. E. Van Deman, the noted pomologist, declares it is one of the best varieties he has ever seen. We offer 5 of these plants and Green's book on fruit growing with each
$\$ 5.00$ to $\$ 10.00$ order and Io plants and Green's book with each $\$ 10.00$ (or over) order, if order is received on or before March I5, with cash. 